\title{
INTRODUCTION À LA CONJECTURE ETNC ET APPLICATIONS
}

\author{
par
}

Cornelius Greither

\begin{abstract}
Résumé. - Ces notes de cours sont une version plus détaillée et complète d'un mini-cours donné aux Journées Arithmétiques de Meknès en automne 2010. Elles ont pour but de décrire l'énoncé de la conjecture ETNC (Equivariant Tamagawa Number Conjecture) dans un cadre bien précis. On traite en détail un exemple non-trivial et on donne une application de la conjecture ETNC, concernant les idéaux de Fitting attachés aux groupes de classes, avec quelques indications sur la démonstration. Souhaitant nous adresser à un large public, nous présentons tous les préliminaires algébriques. A noter que la conjecture ETNC peut être formulée dans une plus grande généralité et nos lecteurs ne doivent pas s'attendre à une discussion systématique de tous ses aspects divers, ni de son historique.
\end{abstract}

Abstract. - In these lecture notes, which arose from a minicourse given at the Journées Arithmétiques de Meknès in the autumn of 2010, we try to describe the statement of the Equivariant Tamagawa Number Conjecture in a particular setting. We discuss a minimal nontrivial example in great detail, and we also present one application concerning Fitting ideals of class groups, with a sketchy outline of proof. In order to make these notes accessible to a somewhat larger audience, we also included a lot of algebraic preliminaries. The conjecture ETNC can be formulated in great generality, and the reader should not expect a systematic discussion of all its variants, nor of its historical origins.

\section{Introduction}

Ce texte, qui essaie de décrire l'énoncé et une application principale de la conjecture ETNC dans un cadre bien précis, a été rédigé pour les Journées Arithmétiques de Meknès (25 - 29 octobre 2010), qui étaient consacrées aux fonctions $L$ en arithmétique. Il a servi de base pour le "mini-cours" (deux exposés de 90 minutes chacun) donné par l'auteur dans ce cadre; beaucoup de détail ont été omis lors des exposés oraux. Après cette réunion, l'auteur a fait quelques corrections et quelques ajouts, mais le texte demeure assez proche du contenu et du style des exposés oraux. Certainement, il ne se veut pas une introduction complète et systématique. En particulier, la formulation de ETNC en général, et son domaine d'application, sont beaucoup

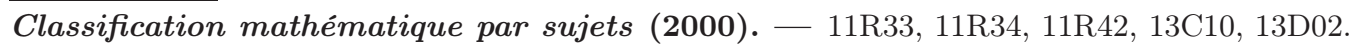

Mots clefs. - Galois modules, L-functions cohomology, projective modules, regulators, class groups, unit groups, Fitting ideals. 
plus vastes qu'on pourrait le penser en regardant notre texte. On n'essaiera pas de retracer les origines de la conjecture, et on sera très bref en ce qui concerne les résultats connus sur sa validité, même dans notre cadre restreint. Notre texte contient des raisonnements élémentaires et détaillés d'une part, et des arguments beaucoup moins élémentaires et incomplets d'autre part; l'auteur a voulu attirer aussi quelques non-spécialistes, tout en prenant le risque d'ennuyer les spécialistes dans la première partie. Signalons aussi que la bibliographie est un peu arbitraire et loin d'être complète.

Notons enfin que l'approche adoptée ici, et le sujet tout entier, doivent beaucoup aux travaux de Burns, et particulièrement à son article [Bu01], et profitons de l'occasion pour remercier cordialement les organisateurs des Journées Arithmétiques de leur efficacité et de leur hospitalité si chaleureuse.

\section{Modules sur les anneaux de groupe}

1.1. Généralités. — Soit $G$ toujours un groupe fini, $R$ un anneau commutatif. On pose

$$
R[G]=\left\{\sum_{g \in G} r_{g} g: r_{g} \in R\right\} .
$$

Souvent on écrit simplement $R G$. Cet ensemble devient un anneau par l'addition évidente, et la multiplication induite par celle de $G$; on appelle $R[G]$ l'anneau du groupe $G$.

Les $R[G]$-modules $M$ ont une autre description : il revient au même de dire que $M$ est un $R$-module muni d'une $G$-action $R$-linéaire, encore autrement : muni d'un homomorphisme de groupes $\varphi: G \rightarrow \operatorname{Aut}_{R}(M)$. Quand $\varphi$ est donné, on retrouve la multiplication $R[G]$-scalaire par : $\left(\sum_{g} r_{g} g\right) \cdot m=\sum_{g} r_{g} \varphi(g)(m)$.

Notre exemple principal sera $G=\operatorname{Gal}(L / K)$ où $L / K$ est une extension galoisienne de corps de nombres, $R$ sera souvent l'anneau $\mathbb{Z}$, et $M$ sera "un objet arithmétique attaché à $L$ ". Ces derniers objets ne manquent pas. Citons $M=L$ ou $M=O_{L}$ (des groupes additifs, donc des $\mathbb{Z}$-modules), $M=O_{L}^{\times}=U_{L}$ (les unités : un groupe abélien normalement noté de façon multiplicative, mais quand même un $\mathbb{Z}$-module), $M=\operatorname{cl}(L)=\operatorname{Pic}\left(O_{L}\right)$. Dans tous les cas, la $\mathbb{Z}[G]$-structure provient de l'action naturelle de $G$ sur l'objet.

Attention : si $G$ n'est pas le groupe trivial, alors $\mathbb{Z} G$ n'est jamais intègre (posons $\mathrm{N}_{G}=\sum_{g} g$ : alors $(h-1) \mathrm{N}_{G}=0$ pour tout $h \in G$ ), et même pas un produit d'anneaux de Dedekind. Il existe toujours un idéal $I \subset \mathbb{Z} G$ qui n'est pas localement cyclique. En général, on ne sait pas classifier les $\mathbb{Z} G$-modules de type fini. Il est déjà difficile (ou impossible, ça dépend de $G$ ) de classifier les modules de type fini sans $\mathbb{Z}$-torsion (modules qu'on appelle réseaux).

Exemple 1.1. - Prenons $K=\mathbb{Q}$ et $L=\mathbb{Q}(\sqrt{3})$. Alors $G=\{i d, \sigma\}$ avec $\sigma(\sqrt{3})=-\sqrt{3}$. Le $\mathbb{Q}[G]$-module (additif) $L$ est libre sur un générateur, par exemple sur $1+\sqrt{3}$; chose connue, car ceci revient à dire que cet élément engendre une base normale. Par contre, le réseau $O_{L}$ n'est pas libre sur $\mathbb{Z}[G]$ (voir la suite).

On a $U_{L}=\{ \pm 1\} \cdot \eta^{\mathbb{Z}}$ où $\eta=2-\sqrt{3}$ est une unité fondamentale; $\bar{U}_{L}:=U_{L} /\{ \pm 1\}$ est un réseau et en fait une copie de $\mathbb{Z}$ (attention : $\bar{U}_{L}$ est multiplicatif). L'élément $\sigma$ agit sur $\bar{U}_{L}$ par inversion, et on a donc $\bar{U}_{L} \cong \mathbb{Z}[G] /(1+\sigma)$. 
1.2. Modules projectifs et modules c.t. - Pour l'instant, $A$ est un anneau quelconque (et les modules seront pris à gauche). Rappelons que $M$ est projectif sur $A$ si et seulement si il y a un autre $A$-module $M^{\prime}$ tel que $M \oplus M^{\prime}$ soit libre. Donc tout module libre est projectif, et toute somme directe de modules projectifs est encore projective. Quand $M$ est de présentation finie (ce qui est le cas dans la plus-que-plupart des cas qu'on rencontre), alors $M$ est projectif si et seulement $M$ est localement libre.

Un exemple banal peut être donné comme suit : Si $A=\mathbb{Z} \times \mathbb{Z}$, alors le module $M=\mathbb{Z} \times 0$ est projectif, mais non libre. Les idéaux a non nuls dans les anneaux $O_{L}$ sont plus intéressants : $\mathfrak{a}$ est toujours projectif (de façon équivalente, localement libre de rang un), et libre si et seulement il est principal (= libre). Donc chaque fois que $h_{L}>1$, on rencontre des cas où $\mathfrak{a}$ est projectif mais non libre. Notons qu'on peut montrer l'existence d'un $O_{L}$-isomorphisme $\mathfrak{a} \oplus \mathfrak{a}^{-1} \cong O_{L} \oplus O_{L}$.

Nous aurons besoin d'un minimum de cohomologie des groupes. Pour l'instant, soit $G$ un groupe fini quelconque. Pour tout $\mathbb{Z}[G]$-module $M$ et tout $i \geq 0$ on peut définir un groupe abélien (dit de cohomologie) $\mathrm{H}^{i}(G, M)$; pour $i \leq 0$, on a aussi des groupes "de Tate" $\hat{\mathrm{H}}^{i}(G, M)$. Pour $i>0$ on posera $\hat{\mathrm{H}}^{i}(G, M)=\mathrm{H}^{i}(G, M)$. Nous n'aurons besoin de ces groupes qu'en très basse dimension. Par définition, $\mathrm{H}^{0}(G, M)=M^{G}$ (éléments fixes) et $\hat{\mathrm{H}}^{0}(G, M)=M^{G} / \mathrm{N}_{G} M$, où $\mathrm{N}_{G}=\sum_{g \in G} g$ est l'élément norme. De plus, $\hat{\mathrm{H}}^{-1}(G, M)$ est le quotient $\operatorname{ker}\left(\mathrm{N}_{G}: M \rightarrow\right.$ $M) / I_{G} M$, où $I_{G}$ est le noyau de l'augmentation sur $\mathbb{Z} G$.

Si $G$ est cyclique, engendré par $\sigma$, alors on a une périodicité :

$$
\hat{\mathrm{H}}^{i+2}(G, M) \cong \hat{\mathrm{H}}^{i}(G, M) \quad \forall i \in \mathbb{Z}
$$

En particulier, $\mathrm{H}^{2}(G, M) \cong M^{G} / \mathrm{N}_{G} M$ et $\mathrm{H}^{1}(G, M) \cong \operatorname{ker}\left(\mathrm{N}_{G}: M \rightarrow M\right) /(\sigma-1) M$.

La définition principale dans ce contexte est la suivante :

Définition 1.2. — Un $\mathbb{Z}[G]$-module $M$ est cohomologiquement trivial (c.t.) si pour tout sous-groupe $G^{\prime}$ de $G$ et pour tout $i$ la cohomologie $\hat{\mathrm{H}}^{i}\left(G^{\prime}, M\right)$ est nulle.

Remarque 1.3. - Il existe des résultats qui permettent de se borner à certains sous-groupes $G^{\prime}$ et certaines dimensions $i$ pour la vérification de cette nullité, mais nous n'entrons pas dans les détails.

Exemple 1.4. - On montre facilement, en partant des définitions, que $M=R[G]$ est c.t. pour tout anneau commutatif $R$. Par conséquent, tous les modules $R[G]$-libres et puis tous les modules $R[G]$-projectifs sont c.t. La suite longue en cohomologie montre : si l'on a une suite courte exacte (s.c.e.) $0 \rightarrow A \rightarrow B \rightarrow C \rightarrow 0$, et deux parmi les trois modules sont c.t., alors le troisième l'est aussi. Ceci permet de dire, par exemple, que $(\mathbb{Z} / n)[G]=\mathbb{Z}[G] / n \mathbb{Z}[G]$ est c.t.; mais ce module n'est pas projectif sur $\mathbb{Z}[G]$.

Proposition 1.5. - Soit $R$ parmi les anneaux $\mathbb{Z}, \mathbb{Z}_{p}, O_{L}, O_{L, \mathfrak{p}}$ et $M$ de type fini sur $R[G]$. Alors :

(a) $M$ est c.t. ssi $M$ est isomorphe à un quotient $P / Q$, où $P$ et $Q$ sont $R[G]$-projectifs de type fini.

(b) Si $M$ est sans torsion et c.t., alors $M$ est déjà projectif sur $R[G]$. 
Exemple 1.6 (suite de l'exemple 1.1). - Rappelons $L=\mathbb{Q}(\sqrt{3}), K=\mathbb{Q}$. Alors $L$ (module additif) est c.t., même libre sur $\mathbb{Q}[G]$. Le module $O_{L}$ n'est pas c.t. : les éléments fixes $O_{L}^{G}$ donnent $\mathbb{Z}$, mais $\mathrm{N}_{G} O_{L}=\operatorname{tr}_{L / K}\left(O_{L}\right)=2 \mathbb{Z}$, donc $\hat{\mathrm{H}}^{0}\left(G, O_{L}\right) \cong \mathbb{Z} / 2$ n'est pas nul.

Comme exercice, le lecteur peut vérifier que $\hat{\mathrm{H}}^{0}\left(G, \bar{U}_{L}\right)=0$ mais $\hat{\mathrm{H}}^{1}\left(G, \bar{U}_{L}\right) \cong \mathbb{Z} / 2$, donc le module $\bar{U}_{L}$ n'est pas c.t. non plus.

1.3. Un exemple classique. - Posons $K=\mathbb{Q}$ et $L=\mathbb{Q}\left(\zeta_{p}\right)$ pour un nombre premier $p>2$ quelconque. Alors $G=\left\{\sigma_{a} \mid a \in(\mathbb{Z} / p)^{\times}\right\} \cong(\mathbb{Z} / p)^{\times}$. Soit $j=\sigma_{-1} \in G$; c'est la conjugaison complexe sur $L$.

Rappelons encore quelques notations connues depuis Kummer : $\mathbb{Q}\left(\zeta_{p}\right)^{+}$désigne le sous-corps réel maximal de $\mathbb{Q}\left(\zeta_{p}\right) ; h_{p}$ est l'ordre de $c l\left(\mathbb{Q}\left(\zeta_{p}\right)\right.$ ) (le nombre de classes), et de même $h_{p}^{+}=$ $\left|c l\left(\mathbb{Q}\left(\zeta_{p}\right)^{+}\right)\right|$. Pour tout module $M$ sur lequel $j$ agit, posons

$$
\begin{aligned}
& M^{+}=\{x \in M: j x=x\}, \\
& M^{-}=\{x \in M: j x=-x\}
\end{aligned}
$$

(parties plus et moins de $M)$. Alors on sait que l'ordre de $\operatorname{cl}\left(\mathbb{Q}\left(\zeta_{p}\right)\right)^{-}$est donné par $h_{p} / h_{p}^{+}=$: $h_{p}^{-}$. En particulier $h_{p}^{+}$divise $h_{p}$.

On s'intéresse à la structure de $C:=\operatorname{cl}\left(\mathbb{Q}\left(\zeta_{p}\right)\right)^{-}$sur $\mathbb{Z}[G]$, ou bien sur l'anneau $A:=\mathbb{Z}[G] /(1+$ $j)$. On commence en cherchant des éléments qui annulent le module fini $C$. On en trouve, en utilisant le célèbre théorème de Stickelberger (1870) (en fait l'énoncé ci-dessous, pour les corps de conducteur premier, était déjà connu de Kummer) :

Théorème 1.7. - Soit $\vartheta=p^{-1} \cdot \sum_{a=1}^{p-1} a \sigma_{a}^{-1} \in \mathbb{Q}[G]$ et $I=\mathbb{Z}[G] \cap \vartheta \mathbb{Z}[G]$ (un idéal dans $\mathbb{Z}[G])$. Alors :

(a) $I \cdot C=0$.

(b) Soit $\bar{I}$ l'image de $I$ dans $A$. Alors $|A / \bar{I}|=h_{p}^{-}$, à un facteur puissance de 2 près.

Pour mieux voir ce qui se passe, on localise (complète) tout en un premier $\ell$ distinct de 2 et de $p$. Alors $A_{\ell}=\mathbb{Z}_{\ell}[G] /(1+j), C_{\ell}$ est la partie $\ell$-primaire $C\{\ell\}$ de $C$, et $\bar{I}_{\ell}=A_{\ell} \vartheta$ est un idéal principal. Ceci laisse penser que

$$
C\{\ell\} \cong A_{\ell} / \vartheta A_{\ell}
$$

Ceci n'est pas vrai en général. (Un contre-exemple est fourni par $\ell=3$ et $p=3299$ : ici $C\{3\}$ n'est pas cyclique sur $A_{3}$.) Il s'agit donc de trouver l'approximation optimale qui soit toujours vraie. L'énoncé $|C\{\ell\}|=\left|A_{\ell} / \vartheta A_{\ell}\right|$ est bien vrai (voir ci-haut) mais trop faible. Ceci se voit bien dans le résultat suivant de Schoof [Sch].

Théorème 1.8. - (a) Le module $C$ est c.t. $\left(\right.$ sur le groupe $\left.G=\operatorname{Gal}\left(\mathbb{Q}\left(\zeta_{p}\right) / \mathbb{Q}\right)\right)$.

(b) Plus concrètement : Rappelons que $A=\mathbb{Z}[G] /(1+j)$. Alors on a une suite courte exacte

$$
0 \rightarrow Q \rightarrow P \rightarrow C \rightarrow 0
$$

où $P$ et $Q$ sont projectifs de type fini sur $A$. (On peut choisir $P$ libre. De plus, pour tout épimorphisme $P \rightarrow C$ avec $P$ projectif, le noyau $Q$ est automatiquement projectif lui aussi.)

Trouvons alors un invariant aussi fin que possible pour les modules finis de la forme $F / Q(F$ libre, $Q$ projectif). Si $Q$ est aussi libre, ce sera encore plus facile. 
1.4. Idéaux de Fitting. — Soit maintenant $A$ un anneau commutatif. Tous les modules seront supposés de présentation finie. Un peu de langage : un 1-complexe $C$ est la donnée de deux $A$-modules $M, N$ et d'un $A$-homomorphisme $\rho: M \rightarrow N$. Si $C$ est de la forme $A^{m} \stackrel{\rho}{\rightarrow} A^{n}$ (i.e., les modules pertinents sont libres), alors on pose

$$
\chi(C):=\langle\operatorname{det}(\sigma): \sigma \text { sous-matrice de format } n \times n \text { de } \rho\rangle_{A} .
$$

Dans le cas spécial $m=n$, cette définition devient beaucoup plus simple : $\chi(C)$ est l'idéal principal de $A$ engendré par le déterminant de $\rho$.

Définition 1.9. - Pour tout $A$-module $M$, on pose $\operatorname{Fitt}_{A}(M)=\chi(C)$, où $C: A^{m} \stackrel{\rho}{\rightarrow} A^{n}$ est tel que $\operatorname{coker}(\rho) \cong M$. (On appelera $C$ une présentation de $M$.)

Bien sûr il faut montrer que l'idéal de Fitting Fitt $_{A}(M)$ est bien défini, c'est-à-dire, ne dépend pas du choix de $C$. Nous omettrons cette vérification.

Faisons un exemple simple avec $A=\mathbb{Z}:$ Si $M=\mathbb{Z} / 5 \times \mathbb{Z} / 10$, on peut prendre $m=n=2$ et $\rho=\left(\begin{array}{cc}5 & 0 \\ 0 & 10\end{array}\right), \operatorname{donc}^{\operatorname{Fitt}_{\mathbb{Z}}}(M)=50 \mathbb{Z}$.

Proposition 1.10. - Soit $A=O[G]$ avec $G$ un groupe abélien fini et $O$ un anneau de Dedekind; soit $M$ un $A$-module qui est torsion en tant que $O$-module. Alors Fitt $_{A}(M)$ est un idéal projectif si et seulement si $M$ est c.t.

Remarque 1.11. - Ce résultat vaut pour tout anneau $A$ noethérien sans nilpotents, si on interprète la condition c.t. dans le sens de la proposition 1.5 a).

Donnons quelques idées de la démonstration de la proposition. Soit $M$ c.t. et de torsion sur $O$. On peut supposer que $A$ est local (pour ce faire, il faut se placer dans le cadre plus général), et on prend une présentation $0 \rightarrow B \rightarrow A^{n} \rightarrow M \rightarrow 0$. Alors $B$ doit être projectif, donc libre : $K=A^{m}$. La condition de torsion force que $m=n$. Donc $M$ est le conoyau de $\rho: A^{n} \rightarrow A^{n}$ et $\operatorname{Fitt}_{A}(M)$ est principal, engendré par $\operatorname{det}(\rho)$. QED

Soit maintenant $G=\operatorname{Gal}\left(\mathbb{Q}\left(\zeta_{p}\right) / \mathbb{Q}\right)$ et $A^{\prime}=\mathbb{Z}[1 / 2][G] /(1+j)$. Soit $C=\operatorname{cl}\left(\mathbb{Q}\left(\zeta_{p}\right)^{-}\right.$et $C^{\prime}=$ $\mathbb{Z}[1 / 2] \otimes_{\mathbb{Z}} C$ (on enlève la partie 2-primaire de $C$ ). Schoof (voir $[\mathbf{S c h}]$ ) a démontré en 1996 :

Théorème 1.12. - Avec ces notations on a

$$
\operatorname{Fitt}_{A^{\prime}}\left(C^{\prime}\right)=I A^{\prime}
$$

où I désigne l'idéal de Stickelberger.

Remarque 1.13. - On peut aussi traiter la 2-partie (plus précisément, démontrer un énoncé sur l'anneau $A=\mathbb{Z}[G] /(1+j)$ et pour $C$ à la place de $\left.C^{\prime}\right)$. C'est en fait couvert dans l'article de Schoof, qui s'appuie sur le travail [Gr92]. Il faut modifier $I$ un peu.

Présentons un modeste exemple numérique.

Posons $p=23$. Calculons $A_{3} / I_{3} ;$ l'idéal $I_{3}$ est principal, engendré par $\vartheta^{\prime}=\sum_{a=1}^{22} a \sigma_{a}^{-1}$ dans $A$ (le dénominateur 23 de $\vartheta$ est inversible dans $A_{3}$ ). Considérons l'homomorphisme 
d'anneaux $f: A \rightarrow \mathbb{Z}_{3}$ donné par $f\left(\sigma_{a}\right)=\left(\frac{a}{23}\right)$ (symbole de Legendre). Nous allons vérifier que $f\left(I_{3}\right)=3 \mathbb{Z}_{3}$ :

$$
\begin{aligned}
f\left(\vartheta^{\prime}\right) & =\sum_{a=1}^{22}\left(\frac{a}{23}\right) \cdot a \\
& =\sum_{a=1}^{11}\left(\frac{a}{23}\right) \cdot(a-(23-a)) \\
& =-21-19-17-15+13+11+9-7-5+3+1 \\
& =-69 .
\end{aligned}
$$

Ceci donne au moins un épimorphisme $A_{3} / I_{3} \rightarrow \mathbb{Z}_{3} / 69 \mathbb{Z}_{3}=\mathbb{Z} / 3 \mathbb{Z}$. En fait c'est un isomorphisme (preuve de l'injectivité omise). On peut même montrer que $A / I^{\prime}=\mathbb{Z} / 3 \mathbb{Z}$, où $I^{\prime}$ est la modification nécessaire pour que tout fonctionne aussi dans la partie 2-primaire (voir la remarque qui précède).

Dans notre cas, $C=\operatorname{cl}\left(\mathbb{Q}\left(\zeta_{23}\right)\right)^{-}=\operatorname{cl}\left(\mathbb{Q}\left(\zeta_{23}\right)\right) \cong \mathbb{Z} / 3 \mathbb{Z}$. Puisque ce module est cyclique et annulé par $I^{\prime}$ (et par $1+j$ ), et que l'indice de $I^{\prime}$ dans $A$ vaut 3 , il est isomorphe à $A / I^{\prime}$. Ceci entraîne directement que $\operatorname{Fitt}_{A}(C)=I^{\prime}$, et on a donc vérifié (en partie) l'énoncé du théorème. Aussi dans la version qui inclut la partie 2-adique, l'idéal (modifié) de Stickelberger est d'indice 3. Remarquons qu'on peut montrer par calculs locaux que $A$ contient exactement un idéal d'indice 3.

Terminons en regroupant quelques propriétés des idéaux de Fitting, qui sont faciles à démontrer. Soit $A$ un anneau commutatif quelconque.

(1) $\operatorname{Fitt}_{A}(A / J)=J$ pour tout idéal $J$. (On vient d'utiliser ceci.)

(2) $\operatorname{Fitt}_{A}(M \oplus N)=\operatorname{Fitt}_{A}(M) \cdot \operatorname{Fitt}_{A}(N)$. (Bon exercice.)

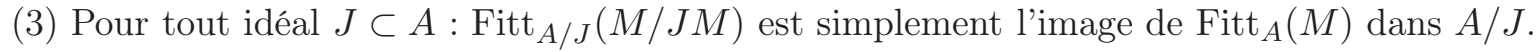

(Changement de base)

\section{2. Énoncé de la conjecture ETNC}

2.1. Régulateurs et fonctions L. - Soit $K$ un corps de nombres, $S \supset S_{\infty}$ un ensemble fini de places de $K,|\cdot|_{v}$ la valeur absolue normalisée qui appartient à la place $v \in S$. On dénote par $U_{S}(K)$ ou par $U_{S}$ le groupe des $S$-unités dans $K$. Soit $Y_{S}$ le $\mathbb{Z}$-module libre de base $S$ et $X_{S} \subset Y_{S}$ le noyau de l'application "somme" (qui envoie tout élément de la base sur 1). On dispose d'une application classique, utilisée déjà par Dirichlet :

$$
\operatorname{reg}_{S}: U_{S} \rightarrow \mathbb{R} \otimes_{\mathbb{Z}} Y_{S}, \quad, u \mapsto-\sum_{v \in S} \log |u|_{v} v .
$$

Citons deux faits importants : l'image de $\operatorname{reg}_{S}$ est contenue dans $\mathbb{R} \otimes X_{S}$ (ceci provient de la formule du produit et de la normalisation des valeurs absolues), et l'image de reg est un réseau dans $\mathbb{R} \otimes X_{S}$, autrement dit : l'application induite

$$
\operatorname{reg}_{S, \mathbb{R}}: \mathbb{R} \otimes_{\mathbb{Z}} U_{S} \rightarrow \mathbb{R} \otimes_{\mathbb{Z}} X_{S}
$$

est un isomorphisme. Ceci provient de la démonstration du théorème de Dirichlet. 
Dorénavant, le rôle de $K$ sera pris par $L$ où $L / K$ est une extension $G$-galoisienne; quand même, $S$ sera toujours attaché à $K$, et l'on aura aussi affaire à $S(L)$, l'ensemble des places de $L$ qui se trouvent au-dessus des places de $S$. Dans cette situation, $U_{S(L)}(L)$ et $X_{S(L)}$ sont munis d'une $G$-action, et l'application $\operatorname{reg}_{S}$ est $\mathbb{Z}[G]$-linéaire.

On part de l'idée générale (pas tout à fait exacte) que la valeur de regs sur une "unite spéciale" doit être donnée par une dérivee d'une fonction $L$ en $s=0$. En fait, plus tard il faudra préciser l'ordre de la dérivée! Le mot-clé est "leading term" = terme dominant. De plus, il ne suffit pas de parler d'une seule fonction $L$ (dans le sens traditionnel), il faut les assembler.

Pour simplifier mettons que $G$ est abélien; soit $\hat{G}$ son groupe de caractères. On a un isomorphisme d'anneaux

$$
\begin{aligned}
\iota: \mathbb{C}[G] & \rightarrow \mathbb{C} \times \cdots \times \mathbb{C}=\mathbb{C}^{\hat{G}}, \\
z & \mapsto(\chi(z))_{\chi \in \hat{G}} .
\end{aligned}
$$

Nous supposerons toujours que $S$ contient $S_{\infty}$ et aussi $S_{\text {ram }}=S_{\text {ram }}(L / K)$, l'ensemble des places de $K$ qui se ramifient dans $L$. Pour tout $\chi \in \hat{G}$ on dispose d'une fonction $L_{S}(\chi, s)$, définie par un produit infini convergent pour $\operatorname{Re}(s)>1$ et admettant une continuation méromorphe en $s$ sur $\mathbb{C}$. En fait cette continuation est holomorphe, car $S \backslash S_{\infty}$ n'est pas vide. On assemble ces fonctions $L$ en une seule, qui prend ses valeurs dans l'anneau de groupe, et qu'on appelle la fonction $L$ équivariante.

Définition 2.1. - Soit $\Theta_{S}(s): \mathbb{C} \rightarrow \mathbb{C}[G]$ la fonction déterminée par

$$
\chi\left(\Theta_{S}(s)\right)=L_{S}\left(\chi^{-1}, s\right)
$$

pour tout $\chi \in \hat{G}$ et pour tout $s \in \mathbb{C}$. Pour un entier positif $k$, la $k$-ième dérivée $\Theta_{S}^{(k)}$ est définie par une formule analogue (remplacer $L_{S}$ par $\left.L_{S}^{(k)}\right)$.

Regardons notre exemple standard $K=\mathbb{Q}, L=\mathbb{Q}\left(\zeta_{p}\right)$, avec $S=\{p, \infty\}$. L'anneau $\mathbb{C}[G]$ s'écrit comme $\mathbb{C}[G]^{+} \oplus \mathbb{C}[G]^{-}$, où $\mathbb{C}[G]^{+}$(respectivement $\mathbb{C}[G]^{-}$) est le noyau de $1-j($ de $1+j$ ) sur $\mathbb{C}[G]$; sous l'isomorphisme $\iota$, la partie $\mathbb{C}[G]^{+}$correspond aux caractères pairs $(\chi(j)=1)$ et l'autre correspond aux caractères impairs $(\chi(j)=-1)$.

On sait que $L_{S}(\chi, s)$ ne s'annule pas en $s=0$ pour $\chi$ impair; par contre, $L_{S}(\chi, s)$ a un zéro d'ordre un en $s=0$ quand $\chi$ est pair. En d'autres termes, la partie plus de $\Theta_{S}(0)$ est nulle, la partie moins ne l'est pas. Ce comportement différent correspond en quelque sens au fait que les unités ont tendance à être concentrées dans la partie plus, c'est-à-dire, dans le sous-corps $L^{+}$maximal réel.

L'élément $\vartheta$ de Stickelberger (voir $§ 1$ ) a une interprétation en termes de la fonction $L$ équivariante. Retenons $S=\{p, \infty\}$. Alors

$$
\Theta_{S}(0)=-\vartheta+\frac{1}{2} \mathrm{~N}_{G} \in \mathbb{Q}[G]^{-} .
$$

Ceci confirme que la partie plus de $\Theta_{S}(0)$ est nulle. Mais il y a un beau lien entre la dérivée $\Theta_{S}^{\prime}(0)$ et les unités cyclotomiques. La formulation originale (en $s=1$, ce qui évite les dérivées) est un peu compliquée. Moyennant l'équation fonctionnelle, on a trouvé une formulation en $s=0$.

Posons $G^{+}=\operatorname{Gal}\left(\mathbb{Q}\left(\zeta_{p}\right)^{+} / \mathbb{Q}\right)$. Il faut choisir une place $w$ de $L^{+}=\mathbb{Q}\left(\zeta_{p}\right)^{+}$au-dessus de $\infty$ (en d'autres termes, il faut fixer un plongement de ce corps dans $\mathbb{R}$ ). On a des identifications 
$X_{S\left(L^{+}\right)} \cong Y_{S_{\infty}\left(L^{+}\right)} \cong \mathbb{Z}\left[G^{+}\right]$, la deuxième donnée par $g w \mapsto g$. (Attention : si l'on considère $w$ comme induit par le plongement $\varphi$ dans $\mathbb{R}$, alors $g w$ est induit par $\varphi g^{-1}$; c'est comme ça, la $G$-action sur les places infinies.) Donc reg $_{S}$ prendra ses valeurs dans $\mathbb{R}\left[G^{+}\right]$maintenant.

Finalement nous avons besoin de l'élément $\lambda^{+}=\left(1-\zeta_{p}\right)\left(1-\zeta_{p}^{-1}\right)$ de $L^{+}$. Ce n'est pas une unité, mais une $p$-unité, donc une $S$-unité, et elle est, à peu près, un générateur du module galoisien des $p$-unités cyclotomiques. Le lien avec la fonction $L$ est le suivant :

Proposition 2.2. -

$$
\Theta_{S}^{\prime}(0)=\frac{1}{2} \operatorname{reg}_{S}\left(\lambda^{+}\right)
$$

C'est en généralisant (de façon conjecturale) cette formule qu'on arrive aux unités de Stark.

2.2. Unités de Stark. - Pour gagner du temps, on se borne au cas de rang 1 (" $r=1$ ", pour les experts). Supposons que $|S| \geq 2$ et que $S$ contient au moins une place $v$ qui est totalement décomposée en $L$. Ceci (avec le choix d'un $w \mid v$ ) fournit une surjection $\pi: X_{S(L)} \rightarrow \mathbb{Z}[G]$. Soit $w_{L}$ le nombre de racines de l'unité contenues dans $L$.

Conjecture Il existe $\eta \in U_{S}(L)$ telle que $\pi \operatorname{reg}_{S}(\eta)=w_{L} \Theta_{S}^{\prime}(0)$.

Il y a aussi un certain énoncé d'unicité dans la conjecture, que nous n'expliquerons pas. L'élément $\eta$ est appelé unité de Stark pour $L / K$ (s'il existe).

Exemple 2.3. $-\lambda^{+}$est une unité de Stark pour $\mathbb{Q}\left(\zeta_{p}\right)^{+} / \mathbb{Q}$.

Pour les groupes de classes dans la partie plus, il n'y a pas d'expression directe en termes de fonctions $L$. Par contre il y a un lien avec ces fonctions quand même, par l'intermédiaire de Stark. C'est largement conjectural, mais citons un cas où c'est établi (voir [CoGr]) :

Théorème 2.4. - Soit $L^{+}=\mathbb{Q}\left(\zeta_{p}\right)^{+}, S=\{p, \infty\}$ et $G^{+}=\operatorname{Gal}\left(L^{+} / \mathbb{Q}\right)$. Alors

$$
\operatorname{Fitt}_{\mathbb{Z}\left[G^{+}\right]}\left(c l\left(L^{+}\right)\right)=\operatorname{Fitt}_{\mathbb{Z}\left[G^{+}\right]}\left(U_{S}\left(L^{+}\right) /\left\langle\lambda^{+}\right\rangle\right) .
$$

On se trouve donc dans la situation suivante : Si l'on se place dans la partie moins (en d'autres termes, si l'on élimine les unités), on trouve (au moins localement) des 1-complexes $\rho: F \rightarrow F$ dont le $\chi$ est donné par $\Theta(0)$ et dont le conoyau est $\mathrm{cl}^{-}$. Si l'on se place dans la partie plus, on trouve une application régulateur partant des unités vers un module explicite $\mathbb{R} \otimes X$; cette fois-ci, les modules ne sont pas projectifs, et $\Theta^{\prime}(0)$ est lié à l'image de cette application. Le groupe de classes dans la partie plus joue toujours un rôle, mais c'est moins visible. Dans uns sens, ETNC incorpore ces deux phénomènes. Pour la suite, il nous faut un certain minimum de technologie.

2.3. Extensions, suites métrisées. - On travaille sur un anneau $\mathfrak{A}$ qui est de type fini comme $\mathbb{Z}$-module, ou comme $\mathbb{Z}_{p}$-module. (Exemples : $\mathbb{Z}[G], \mathbb{Z}[G] /(1+j), \mathbb{Z}_{p}[G]$.) Il est incontournable de rappeler quelques faits de base sur les extensions de modules. Fixons deux $\mathfrak{A}$-modules $M$ et $N$. Sur l'ensemble de toutes les suites courtes exactes (s.c.e.) ayant $N$ (!) à gauche et $M$ à droite, on impose une relation d'équivalence : la ligne supérieure est dite 
équivalente à celle inférieure ssi l'on peut compléter l'échelle ci-bas de façon commutative avec une flèche $E \rightarrow E^{\prime}$ (qui est d'ailleurs automatiquement un isomorphisme).

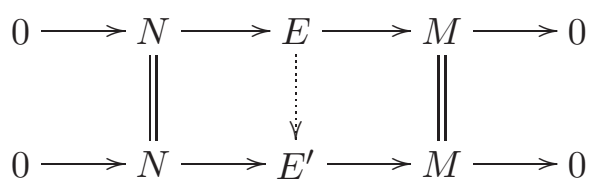

L'ensemble des classes sous cette relation s'écrit $\operatorname{Ext}_{\mathfrak{A}}^{1}(M, N)$, et il est muni d'une structure naturelle de groupe abélien. L'élément neutre est fourni par l'extension scindée : $0 \rightarrow N \rightarrow$ $N \oplus M \rightarrow M \rightarrow 0$.

Pour donner une idée, mentionnons que $\operatorname{Ext}_{\mathbb{Z}}^{1}(\mathbb{Z} / m, \mathbb{Z} / n)$ est cyclique d'ordre pgcd $(m, n)$. En particulier si $m$ et $n$ sont copremiers, toute s.c.e. $0 \rightarrow \mathbb{Z} / n \rightarrow E \rightarrow \mathbb{Z} / m \rightarrow 0$ est scindée. Pour $m=n=2$ on voit bien une suite non scindée, avec $\mathbb{Z} / 4$ au milieu. Elle représente le seul élément non trivial de $\operatorname{Ext}_{\mathbb{Z}}^{1}(\mathbb{Z} / 2, \mathbb{Z} / 2)$.

Il existe des groupes $\operatorname{Ext}_{\mathfrak{A}}^{k}(M, N)$ pour tout $k \geq 1$. Le cas $k=2$ peut être motivé par la concaténation de deux s.c.e. : de $0 \rightarrow Q \rightarrow E_{1} \rightarrow N \rightarrow 0$ et $0 \rightarrow N \rightarrow E_{2} \rightarrow M \rightarrow 0$ on obtient $0 \rightarrow Q \rightarrow E_{1} \rightarrow E_{2} \rightarrow M \rightarrow 0$, où l'application $E_{1} \rightarrow E_{2}$ est, évidemment, la composée $E_{1} \rightarrow N \rightarrow E_{2}$. Sur ces suites exactes à quatre termes (appelés 2-extensions - on ne compte pas les zéros, et on veut $Q$ à gauche et $M$ à droite), on a de nouveau une relation d'équivalence, et un groupe abélien $\operatorname{Ext}_{\mathfrak{A}}^{2}(M, Q)$ qui en résulte. L'élément neutre est, cette fois-ci, donné par

(L'auriez-vous deviné?)

$$
0 \rightarrow Q \stackrel{\equiv}{\rightarrow} Q \stackrel{0}{\rightarrow} M \stackrel{\equiv}{\rightarrow} M \rightarrow 0
$$

On n'a pas le temps de développer la théorie. Citons plutôt une 2-extension naturelle et non triviale sur l'anneau de groupe $\mathbb{Z}[G]$ d'un groupe cyclique $G=\langle\sigma\rangle$ :

$$
0 \rightarrow \mathbb{Z}^{\stackrel{N_{G}}{\rightarrow}} \mathbb{Z}[G] \stackrel{\sigma-1}{\rightarrow} \mathbb{Z}[G] \stackrel{\epsilon}{\rightarrow} \mathbb{Z} \rightarrow 0 .
$$

Ceci fournit un élément $x \in \operatorname{Ext}_{\mathbb{Z}[G]}^{2}(\mathbb{Z}, \mathbb{Z}) \cong \mathrm{H}^{2}(G, \mathbb{Z}) \cong \hat{\mathrm{H}}^{0}(G, \mathbb{Z})$. Ce dernier groupe est égal à $\mathbb{Z} /|G| \mathbb{Z}$, et on peut montrer que $x$ en est un générateur. En des mots vagues : la 2-extension ci-dessus est "aussi non-triviale que possible".

Encore un ingrédient va entrer en jeu : la "suite de Tate". Reprenons la situation : $L / K$ est $G$-galoisienne, $S$ est admissible (i.e., $S$ contient $S_{\infty} \cup S_{\text {ram }}$, et $\operatorname{cl}(L)$ est engendrée par les (classes des) idéaux premiers correspondant aux places finies dans $S(L)$ ).

Alors Tate [Ta] a démontré ce qui suit :

Théorème 2.5. - Il existe une 2-extension de $\mathbb{Z}[G]$-modules

$$
0 \rightarrow U_{S}(L) \rightarrow A \rightarrow B \rightarrow X_{S(L)} \rightarrow 0
$$

telle que $A$ est c.t., $B$ est projectif, et la classe de cette extension dans $\operatorname{Ext}_{\mathbb{Z}[G]}^{2}\left(X_{S(L)}, U_{S}(L)\right)$ est la "classe canonique" fournie par les théories locale et globale du corps des classes.

Nous présentons un exemple qui sera instructif, espérons-le. A retenir que c'est toujours un cas relativement simple!

Revenons à la situation $K=\mathbb{Q}, L=\mathbb{Q}\left(\zeta_{p}\right)^{+}$(donc on n'écrit plus $L^{+}$), $G=\operatorname{Gal}(L / \mathbb{Q})$. Choisissons un générateur $\sigma$ de ce groupe cyclique. Imposons encore les hypothèses $p \equiv 3$ 
modulo 4 , et $h_{L}=1$ (ce qui arrive par exemple pour $p=19,23,31, \ldots$ ). Posons $S=\{p, \infty\}$ comme avant et notons que cet ensemble est admissible. Nous allons travailler avec l'idéal $J=\langle 2, \sigma-1\rangle$ de $\mathbb{Z}[G]$. On peut montrer que $J$ est cyclique, engendré par $\sigma+1$. On peut aussi définir $J$ comme noyau de l'épimorphisme évident $\mathbb{Z}[G] \rightarrow \mathbb{Z} / 2$.

Lemme 2.6. - (a) $U_{S}= \pm\left(\lambda^{+}\right)^{J / 2}$.

(b) $U_{S}$ est c.t.

(c) $X_{S(L)}$ est projectif (même libre).

Démonstration. - (a) (Esquisse) On utilise ce qu'on sait sur les unités cyclotomiques; en particulier il est important que l'élément $\left(\lambda^{+}\right)^{\sigma-1}$ a une racine carrée dans $U_{S}(L)$ (même dans $U(L)$ ). Il faut aussi utilier que toute unité est cyclotomique dans notre cas ( $\left.\operatorname{car} h_{L}=1\right)$.

(b) De (a) on obtient une s.c.e. $0 \rightarrow\{ \pm 1\} \rightarrow U_{S} \rightarrow J / 2 \rightarrow 0$. Puisque $J$ est libre, cette suite est scindée sur $\mathbb{Z}[G]$. De plus, le petit module $\{ \pm 1\}$ est isomorphe à $\mathbb{Z} / 2 \cong \mathbb{Z}[G] / J$, donc il est c.t. Par conséquent $U_{S}$ est c.t. (Il aurait suffi de dire que $U_{S}$ se trouve au milieu d'une s.c.e. aux termes extérieurs qui sont c.t.)

(c) On l'a déjà vu : $X_{S(L)} \cong \mathbb{Z}[G]$.

Nous savons alors que $\operatorname{Ext}_{\mathbb{Z}[G]}^{2}\left(X_{S}, U_{S}\right)=0$, le problème d'identifier la classe canonique ne se pose donc pas! Comme suite de Tate on peut prendre

$$
0 \rightarrow U_{S}(L) \rightarrow U_{S}(L) \rightarrow X_{S(L)} \rightarrow X_{S(L)} \rightarrow 0
$$

avec l'application zéro au milieu. A suivre!

On passe maintenant à une définition technique mais très importante. Sur un anneau général, un module $M$ sera appelé c.t. ssi il s'écrit comme quotient de deux modules projectifs.

Définition 2.7. - Une suite métrisée $(E, \varphi)$ sur un anneau $\mathfrak{A}$ consiste en la donnée d'une 2-extension $E: 0 \rightarrow U \rightarrow A \rightarrow B \rightarrow X \rightarrow 0$ de modules de type fini sur $\mathfrak{A}$, tels que $A$ est c.t. et $B$ est projectif, et d'un isomorphisme

$$
\varphi: \mathbb{R} \otimes_{\mathbb{Z}} U \rightarrow \mathbb{R} \otimes_{\mathbb{Z}} X
$$

de modules sur $\mathbb{R} \otimes_{\mathbb{Z}} \mathfrak{A}$.

L'exemple principal, bien sûr, est fourni par les suites de Tate, où l'isomorphisme $\varphi$ est donné par l'application régulateur pertinente. Les deux prochaines étapes seront :

- d'abord, on introduit la "caractéristique d'Euler" $\chi(E, \varphi) \in \mathrm{K}_{0}(\mathbb{Z}[G], \mathbb{R})$ attachée à n'importe quelle suite métrisée;

— et puis on définit un invariant $T \Omega=T \Omega_{L / K, S}$ comme étant la différence $\chi\left(E_{\text {Tate }}, r e g_{S}\right)$ $\partial\left(\Theta_{S}^{*}(0)\right)$. (Les seules choses qui restent à définir sont le "terme dominant en $s=0$ " noté $\Theta_{S}^{*}(0)$, le K-groupe relatif $\mathrm{K}_{0}(\ldots)$ et le symbole $\partial$.)

Après ces deux étapes, l'énoncé de ETNC est très bref! Le voici :

$$
\operatorname{ETNC}(L / K, S): T \Omega_{L / K, S}=0 .
$$

(Attention : pour les groupes $G$ qui admettent des caractères irréductibles symplectiques, on a triché un peu. Mais tous les groupes abéliens et tous les groupes d'ordre impair sont couverts par cette démarche un peu simplifiée.) 
2.4. Détails de l'énoncé; exemple cyclotomique. - On garde toutes les notations précédentes. Le groupe relatif $\mathrm{K}_{0}(\mathbb{Z}[G], \mathbb{R})$ sera aussi traité dans d'autres exposés. Il consiste en l'ensemble de classes de triplets $[A, \varphi, B]$ où $A$ et $B$ sont $\mathbb{Z}[G]$-projectifs et $\varphi: \mathbb{R} \otimes A \rightarrow \mathbb{R} \otimes B$ est un isomorphisme; on n'explicitera pas ici la relation d'équivalence. Si $G$ est abélien, on peut donner une description plus directe :

$$
\mathrm{K}_{0}(\mathbb{Z}[G], \mathbb{R})=\{P \subset \mathbb{R}[G]: P \text { est } \mathbb{Z}[G] \text {-projectif de rang } 1\},
$$

où l'ensemble à droite est un groupe pour la multiplication dans $\mathbb{R}[G]$. (Le neutre est $\mathbb{Z}[G]$.) En termes de triplets, $P$ correspond à $[P, i d, \mathbb{Z}[G]]$.

On a besoin de trois autres aspects.

D'abord, il y a un homomorphisme "bord" $\partial: \mathbb{R}[G]^{\times} \rightarrow \mathrm{K}_{0}(\mathbb{Z}[G], \mathbb{R})$, défini par $\partial(u)=$ $[\mathbb{Z}[G], u, \mathbb{Z}[G]]$ (ou par $\delta(u)=u \mathbb{Z}[G]$ dans la description simplifiée). Cet homomorphisme devient surjectif quand $\mathbb{Z}[G]$ est remplacé par un anneau local.

De plus, il existe une troisième approche du $\mathrm{K}$ relatif. A tout $\mathbb{Z}[G]$-module $C$ qui est fini et c.t., on peut associer un élément $t(C) \in \mathrm{K}_{0}(\mathbb{Z}[G], \mathbb{R})$ par la recette suivante : prendre une présentation $Q \subset P \rightarrow C \rightarrow 0$ avec $P$ (et par conséquent $Q$ ) projectif, et poser $t(C)=[Q, i, P]$ où $i$ désigne l'inclusion. On appelle ceci dévissage, et l'on pourrait en dire plus loin sur $t$.

Finalement on peut aussi définir $[A, \varphi, B] \in \mathrm{K}_{0}(\mathbb{Z}[G], \mathbb{R})$ quand $B$ est projectif mais $A$ est seulement c.t. Si $A_{\text {tors }}$ est c.t., alors $\bar{A}:=A / A_{\text {tors }}$ l'est aussi, donc projectif, et l'on peut poser

$$
[A, \varphi, B]=[\bar{A}, \varphi, B]-t\left(A_{\text {tors }}\right) .
$$

On saute les détails du cas général.

Maintenant nous sommes prêts pour la première étape mentionnée à la fin du numéro qui précède. Soit donc donnée une suite métrisée $(E, \varphi)$; écrivons-la encore une fois. Sans perte, la flèche $U \rightarrow A$ est une inclusion; on abrègera $\mathbb{R} \otimes_{\mathbb{Z}} U$ en $\mathbb{R} U$ etc.

$$
0 \rightarrow U \subset A \stackrel{d}{\rightarrow} B \stackrel{\pi}{\rightarrow} X \rightarrow 0 ; \quad \varphi: \mathbb{R} U \stackrel{\cong}{\rightarrow} \mathbb{R} X .
$$

Pour bricoler un triple dans $\mathrm{K}_{0}(\mathbb{Z}[G], \mathbb{R})$, les modules $A$ et $B$ sont déjà disponibles ; il manque l'isomorphisme allant de $\mathbb{R} A$ vers $\mathbb{R} B$. Le point essentiel est qu'un tel isomorphisme ressort de la suite métrisée, de façon pas trop compliquée. On va construire ce qu'on appelle la "transposée" $\tilde{\varphi}: \mathbb{R} A \rightarrow \mathbb{R} B$ en partant de $\mathbb{R} \otimes_{\mathbb{Z}} d=d_{\mathbb{R}}$ et de $\varphi$.

Puisque $\mathbb{R}[G]$ est semi-simple, on trouve des sous-modules $A_{0} \subset A$ et $B_{0} \subset B$ tels que :

$$
\begin{aligned}
& \mathbb{R} A=\operatorname{ker}\left(d_{\mathbb{R}}\right) \oplus A_{0} ; \\
& \mathbb{R} B=\operatorname{im}\left(d_{\mathbb{R}}\right) \oplus B_{0} .
\end{aligned}
$$

Notons que $\operatorname{ker}(d)=U$. On construit $\tilde{\varphi}$ par composition comme suit :

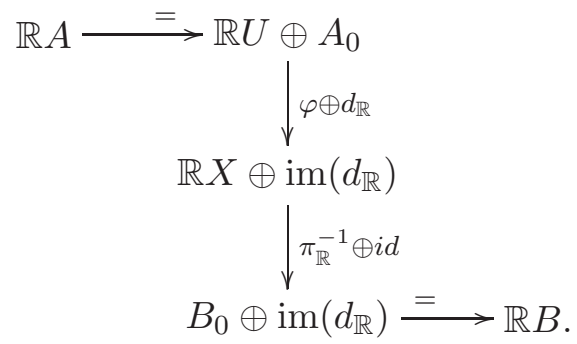


Bien sûr, $\tilde{\varphi}$ dépend des choix qu'on a faits, mais on va voir exactement de quelle façon $\tilde{\varphi}$ peut changer. Regardons l'application $f:=\varphi \oplus d_{\mathbb{R}}$ qui dépend du choix de $A_{0}$. Alors $f$ est compatible avec les deux filtrations $0 \subset \mathbb{R} U \subset \mathbb{R} A$ et $0 \subset \mathbb{R} X \subset \mathbb{R} X \oplus \operatorname{im}\left(d_{\mathbb{R}}\right)$; l'application induite $\mathbb{R} U \rightarrow \mathbb{R} X$ est $\varphi$, et l'application induite $\mathbb{R} A / \mathbb{R} U \rightarrow \operatorname{im}\left(d_{\mathbb{R}}\right)$ est $d_{\mathbb{R}}$. Ceci veut dire que $f$ peut être représenté par blocs :

$$
f=\left(\begin{array}{cc}
\varphi & * \\
0 & d_{\mathbb{R}}
\end{array}\right),
$$

où seulement le bloc $*$ dépend du choix du complément $A_{0}$. Changer $A_{0}$ revient donc à multiplier $f$ par une matrice de la forme $\epsilon=\left(\begin{array}{cc}i d & * \\ 0 & i d\end{array}\right)$ à droite. Cette matrice est élémentaire. De la même façon on trouve qu'un changement de $B_{0}$ va multiplier $\tilde{\varphi}$ par une matrice élémentaire $\epsilon^{\prime}$ à gauche. Il est alors bien connu que les classes $[A, \tilde{\varphi}, B]$ et $\left[A, \epsilon^{\prime} \tilde{\varphi} \epsilon, B\right]$ sont égales dans $\mathrm{K}_{0}(\mathbb{Z}[G], \mathbb{R})$. Donc la classe

$$
\chi(E, \varphi):=[A, \tilde{\varphi}, B]
$$

est bien définie.

Pour l'énoncé final, définissons le terme dominant. Pour n'importe quelle fonction holomorphe non constante $f: \mathbb{C} \rightarrow \mathbb{C}$, soit $k$ l'ordre de $f$ en $s=0$, en d'autres termes : $f(s)=a_{k} s^{k}+$ $a_{k+1} s^{k+1}+\ldots$ avec $a_{k} \neq 0$. On pose $f^{*}(0)=a_{k}$, et l'on définit $\Theta_{S}^{*}(0)$ par $\chi\left(\Theta_{S}^{*}(0)\right)=$ $L_{S}^{*}\left(\chi^{-1}, 0\right)$ pour tout $\chi \in \hat{G}$. (Il est à noter que l'ordre de $L_{S}(\chi, s)$ en $s=0$ peut bien varier avec $\chi$.) Dans un dernier temps, disons que la conjecture qu'on va formuler n'est que ETNC pour un "motif" tout particulier (à savoir, le motif $h^{0}(L)$ ).

Conjecture[ ETNC pour $L / K$ ] Soit $L / K$ une extension $G$-galoisienne (abélienne pour simplifier) de corps de nombres, et soit $S$ un ensemble fini admissible de places de $K$. Soit $E_{\text {Tate }}$ : $0 \rightarrow U_{S}(L) \rightarrow A \rightarrow B \rightarrow X_{S(L)} \rightarrow 0$ une suite de Tate, et soit reg $: \mathbb{R} U_{S}(L) \rightarrow \mathbb{R} X_{S(L)}$ l'isomorphisme régulateur. Alors on a une égalite dans $\mathrm{K}_{0}(\mathbb{Z}[G], \mathbb{R})$ :

$$
\chi\left(E_{\text {Tate }}, \operatorname{reg}_{S}\right)=\partial\left(\Theta_{S}^{*}(0)\right) .
$$

L'expression à gauche est définie comme $\left[A, r \tilde{e g}_{S}, B\right]$, où le tilde indique l'application "transposée" expliquée ci-haut.

Remarquons tout de suite que l'énoncé original est dû à Burns et Flach, et qu'il est, formellement, un peu différent (n'oublions pas que nous nous sommes permis des hypothèses qui simplifient la chose). La conjecture telle qu'énoncée est aussi équivalente à la conjecture LRNC (Lifted Root Number Conjecture) de Ritter et Weiss.

Pour les corps $L$ absolument abéliens, ETNC est démontrée (Burns et G. [BuGr], plus les travaux de Flach pour la partie 2-primaire). De plus, mentionnons les résultats de Bley ([B106] qui traite certaines extensions de corps quadratiques imaginaires) et de Nickel ([Ni10] qui traite la partie moins dans certaines extensions CM, voir aussi la fin du §3.3).

Avant d'essayer de démontrer une conjecture de ce type, on doit toujours faire un certain programme standard, plus précisément : (1) Il faut montrer que la vérité de la conjecture ne dépend pas du choix de $S$ et de la suite de Tate! (2) Il faut établir des résultats de fonctorialité (changement de $L$ ou de $K$ ). Disons au moins un mot sur (1).

L'une des choses à montrer est l'indépendence de $S$ (toujours supposé admissible). Posons qu'il est déjà établi que la caractéristique $\chi\left(E_{\text {Tate }}, r e g_{S}\right)$ ne dépend pas du choix de la suite 
(pourvu toujours qu'il s'agisse d'une suite de Tate; rappelons que les termes $U_{S}(L)$ et $X_{S(L)}$ sont fixés). Il faut donc montrer : Si $S$ est admissible, $S \subset S^{\prime}$, et qu'on a une suite de Tate pour $S$ (les modules étant écrits sans prime), alors on en trouve une (notée avec prime) pour $S^{\prime}$ de sorte qu'on ait une égalité

$$
\left[A^{\prime}, r e \tilde{g}_{S^{\prime}}, B^{\prime}\right]-\left[A, r \tilde{e}_{S}, B\right]=\partial\left(\Theta_{S^{\prime}}^{*}(0) / \Theta_{S}^{*}(0)\right)
$$

Nous ne donnons que quelques indications. Par récurrence on peut supposer que $S^{\prime}=S \cup\left\{v^{*}\right\}$; notons que $v^{*}$ est non ramifié.

On choisira une suite de Tate pour $S^{\prime}$ qui se déduit de celle donnée pour $S$ de façon canonique. Soit $S_{v^{*}}(L)$ l'ensemble des places de $L$ au-dessus de $v^{*}$. On a des isomorphismes naturels $\alpha: U_{S^{\prime}}(L) / U_{S}(L) \cong \mathbb{Z} S_{v^{*}}(L)$ et $\mathbb{Z} S^{\prime}(L) / \mathbb{Z} S(L)=\mathbb{Z} S_{v^{*}}(L)$. Utilisant un argument explicite qui remonte à Chinburg (aujourd'hui beaucoup d'auteurs utilisent le langage des catégories dérivées) on établit une "suite courte exacte de suites métrisées" :

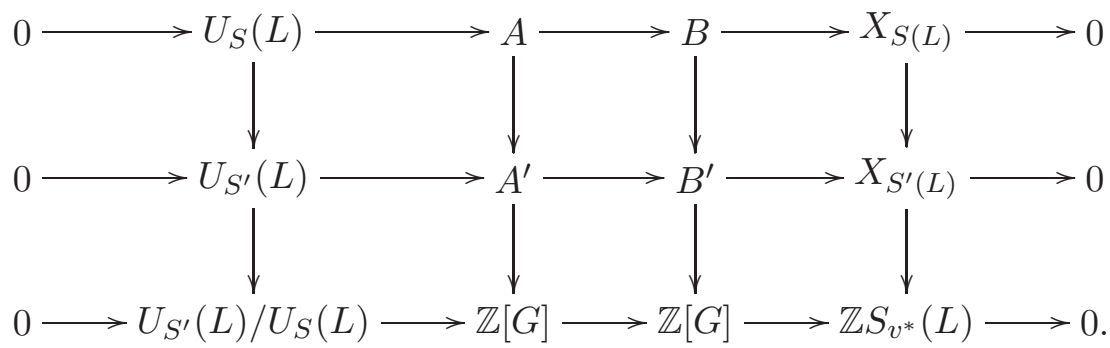

Dans ce diagramme, les trois métrisations (la troisème étant donnée à peu près par $\alpha$ ) sont compatibles, et l'on peut invoquer un résultat de multiplicativité pour $\chi$ [Bu01]. De plus, la suite d'en bas est complètement connue. Si nous supposions pour simplifier que $v^{*}$ est totalement inerte, ce serait exactement la suite citée quelques lignes avant le théorème 2.5 à titre d'exemple simple mais non trivial. De l'autre côté, le quotient $\Theta_{S^{\prime}}^{*}(0) / \Theta_{S}^{*}(0)$ est un facteur eulérien, dont le terme dominant, comme on peut le montrer, correspond exactement au $\chi$ de la troisième suite métrisée.

Faisons la suite de notre exemple cyclotomique. Pour toute la notation, on renvoie au numéro 2.3. La suite de Tate est assez simple :

$$
0 \rightarrow U_{S} \rightarrow U_{S} \stackrel{0}{\rightarrow} X_{S} \rightarrow X_{S} \rightarrow 0 .
$$

On a $U_{S}=\{ \pm 1\} \oplus\left(\lambda^{+}\right)^{J / 2}, X_{S} \cong \mathbb{Z}[G]$, et $\operatorname{reg}_{S}\left(\lambda^{+}\right)=2 \Theta_{S}^{*}(0)$. Puisque la suite de Tate a une application zéro au milieu, la transposée de $r e g_{S}$ est heureusement facile à trouver : elle coïncide avec $\mathrm{reg}_{S}$. On trouve (rappelons que $t(C)$ a été défini au $\S 2.4$, pour tout module $C$ fini et c.t.) :

$$
\begin{aligned}
\chi\left(U_{S}, \text { reg }_{S}, \mathbb{Z}[G]\right) & =\chi\left(U_{S} /\{ \pm 1\}, \text { reg }_{S}, \mathbb{Z}[G]\right)-t(\{ \pm 1\}) \\
& =\chi\left(J / 2,2 \Theta_{S}^{*}(0), \mathbb{Z}[G]\right)-[J, i, \mathbb{Z}[G]] \\
& =\chi\left(J, \Theta_{S}^{*}(0), \mathbb{Z}[G]\right)-[J, i, \mathbb{Z}[G]] \\
& =\chi\left(\mathbb{Z}[G], \Theta_{S}^{*}(0), \mathbb{Z}[G]\right) \\
& =\partial\left(\Theta_{S}^{*}(0)\right) .
\end{aligned}
$$

On a donc confirmé ETNC dans cet exemple. 


\section{Applications et compléments}

3.1. Idéaux de Fitting dans la partie moins. - Soit toujours $L / K$ une extension abélienne, de groupe de Galois $G$, où $K$ est totalement réel et $L$ un corps CM. Pour motiver notre démarche, décrivons quelques constructions et résultats de Kurihara pour $K=\mathbb{Q}$. Le but est de calculer Fitt $_{\mathfrak{A}^{\prime}}\left(\operatorname{cl}(L)^{-}\right)$, où $\mathfrak{A}^{\prime}=\mathbb{Z}[1 / 2][G] /(1+j)$ et par abus de notation $c l(L)^{-}$ dénote la partie moins de $c l(L)$ avec la partie 2-primaire enlevée. Cet abus de notation vaudra pour tous les modules : on tensorise avec $\mathbb{Z}[1 / 2]$ avant de prendre le noyau de $1+j$.

Il est clair que tout résultat dans cette direction impliquera les idéaux de Stickelberger. Mais déjà pour $L=\mathbb{Q}\left(\zeta_{n}\right)$ où $n$ n'est pas la puissance d'un premier, la généralisation immédiate serait trop naïve : il ne suffit pas de poser $\vartheta_{n}=n^{-1} \sum_{(a, n)=1} a \sigma_{a}^{-1}$ et de prendre l'idéal $Z\left[G_{n}\right] \cap \vartheta_{n} \mathbb{Z}\left[G_{n}\right]$. (On a posé $G_{n}=\operatorname{Gal}\left(\mathbb{Q}\left(\zeta_{n}\right) / \mathbb{Q}\right)$.) Même dans la partie moins, l'indice de cet ideal dans $\mathfrak{A}$ sera en général infini.

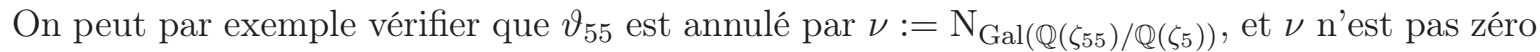
dans $\mathbb{Z}\left[G_{55}\right] /(1+j)$. La sortie de cette impasse a été trouvée par Sinnott (et Kurihara se sert d'une variante) : il faut mettre beaucoup de générateurs, provenant des sous-corps de $L$, dans l'idéal de Stickelberger à construire.

Pour aller plus vite, bornons-nous au cas $L=\mathbb{Q}\left(\zeta_{n}\right)$. Dans ce cas, les constructions de Sinnott et de Kurihara sont les mêmes. Un diviseur distingué de $n$ est par definition un $m \mid n$ tel que $m$ et $n / m$ sont copremiers. Soit $D_{n}$ l'ensemble des diviseurs distingués de $n$. Pour tout $m \mid n$ on a un épimorphisme naturel $G_{n} \rightarrow G_{m}$ et une injection "corestriction" $\operatorname{cor}_{m, n}: \mathbb{Z}\left[G_{m}\right] \rightarrow \mathbb{Z}\left[G_{n}\right]$ qui envoie 1 sur $\mathrm{N}_{\operatorname{ker}\left(G_{n} \rightarrow G_{m}\right)}$. On pose :

$$
\begin{aligned}
I_{n}^{\prime} & =\left\langle\operatorname{cor}_{m, n}\left(\vartheta_{m}\right): m \in D_{n}\right\rangle_{\mathbb{Z}\left[G_{n}\right]} ; \\
I_{\mathbb{Q}\left(\zeta_{n}\right)}^{(K u)} & =\mathbb{Z}\left[G_{n}\right] \cap I_{n}^{\prime} .
\end{aligned}
$$

L'idéal $I_{L}^{(K u) ~ " a ̀ ~ l a ~ K u r i h a r a " ~ e s t ~ p a r ~ c o n t r e ~ d e ́ f i n i ~ p o u r ~ t o u t ~} L$ absolument abélien. Le résultat suivant provient de travaux de Kurihara $[\mathbf{K u 0 3}]$ et de Kurihara et Miura $[\mathbf{K u M i}]$.

Théorème 3.1. - Soit L absolument abélien, de groupe $G$ sur $\mathbb{Q}$. Alors

$$
\operatorname{Fitt}_{\mathfrak{A}^{\prime}}\left(c l(L)^{-}\right)=I_{L}^{(K u)} \mathfrak{A}^{\prime} .
$$

Notre but dans le travail [Gr07] était d'étendre ce résultat aux extensions abéliennes du type $\mathrm{CM}$, c'est-à-dire que $K$ est totalement réel et $L$ est totalement complexe, en supposant que ETNC est vraie. Les méthodes de Kurihara ne sont applicables qu'avec des hypothèses assez restrictives. En fait l'énoncé ne se généralise pas tel quel (voir la suite), il faut le changer quelque peu. Comme point de départ, la construction des idéaux donnée ci-dessus se généralise : on a un idéal $I_{L / K}^{(K u)} \subset \mathbb{Z}[G]$ pour toute extension $L / K G$-abélienne avec $K$ totalement réel et $L$ un corps CM.

Mais quel est le lien avec ETNC? Cette conjecture nous dit beaucoup sur les suites de Tate. Mais le groupe $\operatorname{cl}(L)$ est caché subtilement dans cette suite; il se trouve "dans $U_{S}(L)$ ", en raison de la condition que les $S$-places engendrent $c l(L)$. Un point essentiel est l'existence de suites " de Tate" pour de plus petits ensembles $S$, en particulier pour l'ensemble minimal $S_{\infty}$. Le prix à payer est que le terme à droite (qui est $X_{S}$ jusqu'à maintenant) devient moins beau. Par contre, ce terme à droite contiendra $\operatorname{cl}(L)$ de façon plus visible, et le terme à gauche au 
moins ne devient pas plus difficile. Donnons quelques détails. L'existence et les propriétés des "suites de Tate pour les petits ensembles $S$ " ont été découvertes par Ritter et Weiss.

Notons pour commencer que l'ensemble $S_{\infty}$ des places infinies est rarement admissible. Notons aussi que $U_{S_{\infty}}(L)=U(L)=O_{L}^{\times}$.

La suite pour $S_{\infty}$ prend la forme suivante :

$$
0 \rightarrow U(L) \rightarrow A_{\infty} \rightarrow B_{\infty} \rightarrow \nabla \rightarrow 0 .
$$

La complication principale est le module $\nabla=\nabla_{S_{\infty}}$. C'est un module de type fini sur $\mathbb{Z}[G]$, et on sait que

$$
\nabla_{\text {tors }}=\operatorname{cl}(L)
$$

La "partie sans torsion" $\bar{\nabla}:=\nabla / \nabla_{\text {tors }}$ est plus compliquée à décrire. Elle reflète la ramification dans l'extension $L / K$. Plus concrètement, soit $S$ admissible; on attache un "module de ramification" $W_{v}$ à chaque place finie $v \in S$ (ce module est projectif sur $\mathbb{Z}\left[G_{v}\right]$ ssi $v$ est non ramifié); pour $v$ infini on pose $W_{v}=\mathbb{Z}$. Alors $\bar{\nabla}$ est le noyau d'une application canonique $\bigoplus_{v \in S} \operatorname{ind}_{G_{v}}^{G}\left(W_{v}\right) \rightarrow \mathbb{Z}$. (On voit une analogie avec la définition du module plus simple $X_{S}$.) Le programme pour calculer $\operatorname{Fitt}_{\mathfrak{A}^{\prime}}\left(\mathrm{cl}(L)^{-}\right)$est alors le suivant :

(1) On modifie la suite qui précède comme suit :

$$
\left(E_{\infty}^{-}\right) \quad 0 \rightarrow U(L)^{-} \rightarrow A_{\infty}^{-} \rightarrow B_{\infty}^{\prime}{ }^{-} \rightarrow(\nabla / F)^{-} \rightarrow 0 .
$$

Ici $F$ sera un sous-module libre auxiliaire de $\nabla$ tel que $\nabla / F$ est fini. C'est la première modification; de plus, on prend la partie moins partout. Il est bien connu que $U(L)^{-}$est fini et égal à $\mu_{L}^{\prime}$ (les racines de l'unité de $L$, modulo la partie 2-primaire). Donc cette suite possède une unique métrisation, à savoir : la métrisation triviale $\varphi_{0}$. En utilisant la validité de ETNC, on est en mesure de calculer $\chi\left(E_{\infty}^{-}, \varphi_{0}\right)$.

Puis, on peut établir une métrisation $\varphi_{\infty}$ sur cette suite et calculer $\chi\left(E_{\infty}, \varphi_{\infty}\right)$.

(2) Le module $(\nabla / F)^{-}$est une approximation au module $c l(L)^{-}$; dans cette étape on calcule son idéal de Fitting, appuyé sur la connaissance de $\chi\left(E_{\infty}^{-}, \varphi_{0}\right)$ et un lemme. Noter que si $\mu_{L}^{\prime}=0$, alors la suite $\left(E_{\infty}^{-}\right)$ressemble beaucoup aux présentations qui servent pour définir les idéaux de Fitting.

(3) On fait le lien entre $(\nabla / F)^{-}$et le groupe de classes $\operatorname{cl}(L)^{-}$. Ceci faisant, il arrive quelque chose d'inattendu : les calculs nous contraignent à remplacer $c l(L)^{-}$par son dual (de Pontryaguine).

Voilà le programme. On donnera quelques explications sur (2) et (3) seulement. Supposons pour simplifier que $\mu_{L}^{\prime}$ est trivial. Quant à (2), on a besoin d'un lemme. Rappelons que dans notre cadre, le groupe relatif $K_{0}(\mathbb{Z}[G], \mathbb{R})$ peut aussi être vu comme groupe multiplicatif de tous les idéaux fractionnaires $P \subset \mathbb{Z}[G]$, plus explicitement : $P$ parcourt les $\mathbb{Z}[G]$-sous-modules localement libres de rang un dans $\mathbb{R}[G]$. Rappelons aussi que $\operatorname{Fitt}_{\mathbb{Z}[G]}(N)$ est localement libre de rang un pour tout $N$ qui est fini et c.t.

Lemme 3.2. - Soit $(E): 0 \rightarrow 0 \rightarrow \tilde{A} \stackrel{d}{\rightarrow} \tilde{B} \rightarrow N \rightarrow 0$ une suite métrisée. Alors $N$ est fini, la métrisation $\varphi$ est triviale (notons-la donc $\varphi_{0}$ ), et on a

$$
\chi\left(E, \varphi_{0}\right)=\operatorname{Fitt}_{\mathbb{Z}[G]}(M) .
$$


Démonstration. - La métrisation $\varphi$ est un isomorphisme $\mathbb{R} \otimes 0 \rightarrow \mathbb{R} \otimes N$. Par conséquent, $N$ doit être fini, et $\varphi=\varphi_{0}$. Par construction alors, la transposée $\tilde{\varphi}_{0}$ vaut $d_{\mathbb{R}}$. Donc on a

$$
\chi\left(E, \varphi_{0}\right)=[\tilde{A}, d, \tilde{B}] .
$$

Quand on passe de la description via triplets à l'autre (via modules localemet libres de rang un), on voit que ce triplet correspond exactement à l'idéal de Fitting du conoyau de $d$ (calculer localement).

Soit alors $\chi\left(E_{\infty}^{-}\right)=\partial(f)$. (Comme indiqué plus haut, on "connaît l'élément $f$ "; plus précisément, il est donné par une expression compliquée, dans laquelle le seul ingrédient non explicite est le terme dominant de la fonction L équivariante). Alors les deux lemmes pris ensemble produisent

$$
\operatorname{Fitt}_{\mathfrak{A}^{\prime}}\left((\nabla / F)^{-}\right)=(f) .
$$

Expliquons maintenant l'étape (3). Rappelons que $F$ est un sous-module libre de $\nabla$, que $\nabla_{\text {tors }}=\operatorname{cl}(L)$, et qu'on a posé $\bar{\nabla}=\nabla / \nabla_{\text {tors }}$; alors $F$ peut aussi être vu comme sous-module de $\bar{\nabla}$, et on a une s.c.e.

$$
0 \rightarrow \operatorname{cl}(L)^{-} \rightarrow(\nabla / F)^{-} \rightarrow(\bar{\nabla} / F)^{-} \rightarrow 0 .
$$

Ici on connaît l'idéal de Fitting du terme au milieu (qui est c.t.), et on connaît le terme à droite complètement (on a omis beaucoup de détail ici). Il faut maintenant passer à une suite avec quatre termes. Soit $x \in \mathfrak{A}^{\prime}$ tel que $x \bar{\nabla}^{-} \subset F^{-}$. Alors on obtient

$$
0 \rightarrow c l(L)^{-} \rightarrow(\nabla / F)^{-} \rightarrow x^{-1} F^{-} / F^{-} \rightarrow x^{-1} F^{-} / \bar{\nabla}^{-} \rightarrow 0 .
$$

Ici le troisième et le quatrième terme sont explicites, et le deuxième et le troisième sont c.t. Ceci permet d'appliquer le lemme suivant :

Lemme 3.3. - Soit donné une suite exacte de modules finis sur $\mathfrak{A}^{\prime}$ (ou sur $\mathbb{Z}[G]$ ) de la forme

$$
0 \rightarrow M \rightarrow Q \rightarrow Q^{\prime} \rightarrow M^{\prime} \rightarrow 0
$$

telle que $Q$ et $Q^{\prime}$ sont c.t. Alors on a la formule (Fitt étant pris sur $\mathfrak{A}^{\prime}$ ou sur $\mathbb{Z}[G]$, selon le cas) :

$$
\operatorname{Fitt}\left(M^{\vee}\right)=\operatorname{Fitt}(Q) \cdot \operatorname{Fitt}\left(Q^{\prime}\right)^{-1} \cdot \operatorname{Fitt}\left(M^{\prime}\right) .
$$

Remarques : (a) L'idéal Fitt $\left(Q^{\prime}\right)$ est projectif de rang un, donc l'inversion a bien un sens.

(b) Il est incontournable dans la preuve de prendre le dual $M^{\vee}$ au lieu de $M$.

Quand on applique ce lemme à nos données, on obtient une formule compliquée pour $\operatorname{Fitt}_{\mathfrak{A}^{\prime}}\left(\mathrm{cl}(L)^{-}\right)$. Après des calculs un peu longs on arrive finalement au résultat qui suit [Gr07] :

Théorème 3.4. - Soit $L / K$ une extension abélienne avec groupe $G, K$ totalement réel et $L$ totalement complexe. Supposons que ETNC est vraie pour $L / K$, et que $L$ ne contient pas de racines de l'unité d'ordre impair (ou plus généralement que $\mu_{L}^{\prime}$ est c.t. en tant que G-module). Alors

$$
\operatorname{Fitt}_{\mathfrak{A}^{\prime}}\left(c l(L)^{-\vee}\right)=I_{L / K}^{(K u)} \cdot \mathfrak{A}^{\prime}
$$

où $I_{L / K}^{(K u)}$ est un idéal du type Stickelberger, construit par Kurihara. 
Mentionnons pour terminer qu'il y a une classe d'extensions ("nice extensions") pour lesquelles cet idéal est principal dans la partie moins ; la formule pour $\operatorname{Fitt}_{\mathfrak{A}^{\prime}}\left(\mathrm{cl}(L)^{-\vee}\right)$ était connue sans aucune condition (ETNC ou quoi que ce soit), voir Thm. 4.11 dans [Gr00]. Dans ce cas assez particulier on n'a pas besoin du dual, parce que $c l(L)^{-}$est c.t. pour les extensions appelées "nice", et l'idéal de Fitting d'un module fini c.t. ne change pas sous dualisation. (Mais en général, il change certainement, voir §3.2.) Il est d'ailleurs très intéressant de voir que Nickel a démontré la partie moins de ETNC pour les extensions "nice"; voir [Ni09].

3.2. Résultats négatifs sur l'idéal de Fitting du groupe de classes. - Contentonsnous d'une esquisse :

(a) Dans un article en commun avec Kurihara [GrKu], on démontre que l'énoncé du théorème 3.4 devient faux en général si l'on ne prend pas le dual de $c l(L)^{-}$. En effet, déjà l'inclusion $\supset$ est fausse.

Pour rendre ce phénomène plus transparent, prenons un exemple purement algébrique. Soit $G$ le groupe non cyclique d'ordre 4, avec générateurs $s$ et $t$. On va considérer des modules sur $\mathbb{Z}[G]$ annulés par 2 , donc des modules sur $\mathbb{F}_{2}[G]$. Posons $x=1-s$ et $y=1-t$ dans $\mathbb{F}_{2}[G]$. Alors $x^{2}=y^{2}=0$, et $1, x, y, x y$ sont une $\mathbb{F}_{2}$-base de $\mathbb{F}_{2}[G]$. Soit $M=\mathbb{F}_{2}[G] /(x y)$; il est facile

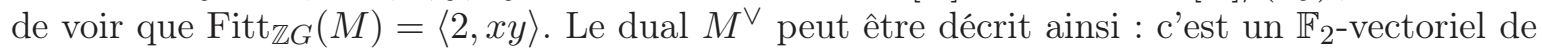
base $u, v, w ; x u=w=y v$, et $y u=x v=0$. Ceci donne une présentation $\operatorname{sur} \mathbb{Z}[G]$ avec deux générateurs $u, v$ et les relations $2 u=2 v=0, y u=x v=0, x u=y v$. Ceci nous donne une présentation de $M^{\vee}$ ayant une matrice

$$
\left(\begin{array}{cc}
2 & 0 \\
0 & 2 \\
y & 0 \\
0 & x \\
y & -x
\end{array}\right)
$$

On peut montrer directement que l'idéal engendré par les sous-déterminants $2 \times 2$ de cette matrice ne contient pas 2. Donc $\operatorname{Fitt}_{\mathbb{Z}[G]}\left(M^{\vee}\right)$ est distinct de (en fait, proprement contenu

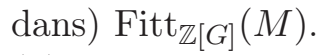

(b) Dans une prépublication très récente [Ku10], Kurihara démontre que même si l'on retient le dual, le théorème 3.4 devient faux à défaut de la trivialité cohomologique de $\mu_{L}$ en dehors de 2. En effet il montre davantage : il existe des exemples pour lesquels on n'a même pas l'inclusion $\supset$ dans le théorème 3.4, qu'on dualise $\mathrm{cl}\left(L^{-}\right)$ou non. Ceci semble indiquer qu'il n'a pas de formule nette pour $\operatorname{Fitt}_{\mathfrak{A}^{\prime}}\left(c l(L)^{-\vee}\right)$ en toute généralité.

Esquissons un exemple donné par Kurihara. Pour $K$ on peut prendre le corps réel biquadratique $\mathbb{Q}(\sqrt{79}, \sqrt{69})$. Le corps quadratique $\mathbb{Q}(\sqrt{79})$, dont le nombre des classes vaut 3 , admet une extension $F_{1}$ cubique cyclique non ramifiée partout, et le corps quadratique $\mathbb{Q}(\sqrt{69})$ admet une extension cubique cyclique $F_{2}$ non ramifiée en dehors de 3 et non absolument abélienne. Par conséquent, $L_{0}=F_{1} F_{2}$ est linéairement disjoint de $k\left(\zeta_{3} \infty\right)$, et $L_{0} / K$ est une extension abélienne de groupe $\mathbb{Z} / 3 \times \mathbb{Z} / 3$. C'est presque l'exemple cherché ; pour le moment, les résultats de Kurihara garantissent au moins que pour $n$ assez grand, l'inclusion de 3.1.5 ne vaudra pas pour $L=L_{0}\left(\zeta_{3^{n}}\right) / K$, avec et sans dualisation. Il est probablement possible de spécifier une valeur explicite de $n$. Notons que le groupe $\mu_{L}$ n'est pas c.t. pourvu que $n>0$. En effet, 
$\operatorname{Gal}(L / K)$ contient un sous-3-groupe non trivial, et celui agit trivialement sur $\mu_{L}\{3\}$, qui par conséquent ne peut être cohomologiquement trivial.

3.3. Suites "simples" de Tate. — Dans cette section finale et extrêmement fragmentaire nous voudrions mentionner des travaux actuels de G. et Popescu [GrPo]. La notion de suite de Tate existe aussi bien pour les "corps de fonctions", plus précisément pour les extensions galoisiennes de corps globaux $L / K$ de caractéristique finie. Un tel corps $K$ (ou $L$ ) est toujours une extension fini d'un corps de fonctions rationnelles $\mathbb{F}(t)$, où $\mathbb{F}$ est un corps fini.

Dans ce cadre, G. et P. ont trouvé une description assez directe des complexes pertinents pour ETNC; cette description revient aux 1-motifs utilisés par Deligne dans sa démonstration de la conjecture de Brumer-Stark (voir le livre [Ta] sur les conjectures de Stark). Ajoutons tout de suite que les 1-motif sont sympathiques et beaucoup plus simples et abordables que les motifs généraux.

Grosso modo, sous certaines hypothèses on peut attacher, pour tout $\ell$, un $\mathbb{Z}_{\ell}[G]$-module $M$ à toute extension $G$-galoisienne $L / K$ de corps de fonctions. (Le nombre premier $\ell$ pourra, ou non, être égal à la caractéristique de $K$.) Ce module $M$ est de type fini, sans torsion, et muni d'une action naturelle de Frobenius (noté $F$ ). G. et P. démontrent que $M$ est c.t., et que $1-F: M \rightarrow M$ donne naissance à une 2-extension $0 \rightarrow \mathbb{Z}_{\ell} U_{S, T} \rightarrow M \rightarrow M \rightarrow \mathbb{Z}_{\ell} X_{S} \rightarrow 0$. Ici, l'ensemble $T$ est une autre donnée technique; c'est un ensemble fini non vide de places de $K$, qui ne rencontre pas $S$. Dans un certain sens on est aussi capable de montrer que cette 2-extension est une suite de Tate. On doit expliquer ici ce qu'on entend par "suite de Tate", car on a changé légérement le terme de départ.

En partant de cette construction relativement simple d'une suite de Tate, on peut vérifier la validité de ETNC. A notre connaissance, c'était établi avant par Burns pour toutes les extensions de corps de fonctions. Mais il semble possible d'étendre la construction au cas des corps de nombres, avec retombées potentielles sur ETNC dans ce cas. En tout cas il faut comparer ceci avec les travaux récents de Nickel (voir en particulier [Ni10]) qui, lui aussi, sait établir la validité de ETNC dans la partie moins pour les corps de nombres sous certaines contraintes.

\section{Références}

[B106] W. Bley, On the equivariant Tamagawa number conjecture for Abelian extensions of a quadratic imaginary field, Doc. Math. 11 (2006), 73-118.

[Bu01] D. Burns, Equivariant Tamagawa numbers and Galois module theory I, Compositio Math. 129 (2001), 203-237.

[BuGr] D. Burns and C. Greither, On the equivariant Tamagaw ; number conjecture for Tate motives, Invent. Math. 153 (2003), 305-359

[CoGr] P. Cornacchia and C. Greither, Fitting ideals of class groups of real fields with prime power conductor, J. Number Theory 73 (1998), 459-471.

[Gr92] Class groups of abelian fields, and the main conjecture, Ann. Inst. Fourier 42 (1992), 449500.

[Gr00] C. Greither, Some cases of Brumer's conjecture for abelian CM extensions of totally real fields, Math. Z. 233 (2000), 515-534. 
[Gr07] C. Greither, Determining Fitting ideals of minus class groups via the equivariant Tamagawa number conjecture, Compos. Math. 143 2007, 1399-1426.

[GrPo] C. Greither and C. Popescu, The Galois module structure of $\ell$-adic realizations of Picard 1-motives and applications, prépublication 2010.

[GrKu] C. Greither and Masato Kurihara, Stickelberger elements, Fitting ideals of class groups of CM-fields, and dualisation, Math. Z. 260 (2008), 905-930.

[Ku03] M. Kurihara, Iwasawa theory and Fitting ideals, J. reine angew. Math. 561 (2003), 39-86.

[KuMi] M. Kurihara and Takashi Miura, Stickelberger ideals and Fitting ideals of class groups for abelian number fields, preprint 2009.

[Ku10] M. Kurihara, Remarks on the Fitting ideals of the duals of ideal class groups, preprint 2010.

[Ni09] A. Nickel, The lifted root number conjecture for small sets of places, J. Lond. Math. Soc. 80 (2009), 446-470.

[Ni10] A. Nickel, On the equivariant Tamagawa number conjecture in tame CM-extensions, to appear in Math. Z., DOI 10.1007/s00209-009-0658-9.

[Sch] René Schoof, Minus class groups of the fields of the l-th roots of unity, Math. Comput. 67 1998, 1225-1245.

[Ta] J. Tate, Les conjectures de Stark pour les fonctions L d'Artin en $s=0$, Progress in Mathematics Vol. 47, Birkhäuser, Boston 1984.

7 décembre 2010

Cornelius Greither, Institut für Theoretische Informatik und Mathematik, Universität der Bundeswehr, München, 85577 Neubiberg, Germany • E-mail : cornelius.greither@unibw.de 